\title{
Synthesis of blue-shifted luminescent colloidal GaN nanocrystals through femtosecond pulsed laser ablation in organic solution
}

\author{
Abdülmelik Demirel • Tuğba Öztaş • \\ Canan Kurşungöz • İbrahim Yılmaz • \\ Bülend Ortaç
}

Received: 6 August 2015/Accepted: 2 May 2016/Published online: 12 May 2016

(C) Springer Science+Business Media Dordrecht 2016

\begin{abstract}
We demonstrate the synthesis of $\mathrm{GaN}$ nanocrystals (NCs) with the sizes of less than the doubled exciton Bohr radius leading quantum confinement effects via a single-step technique. The generation of colloidal GaN nanoparticles (NPs) in organic solution through nanosecond (ns) and femtosecond (fs) pulsed laser ablation (PLA) of GaN powder was carried out. Ns PLA in ethanol and polymer matrix resulted in amorphous GaN-NPs with the size distribution of $12.4 \pm 7.0$ and $6.4 \pm 2.3 \mathrm{~nm}$, respectively, whereas fs PLA in ethanol produced colloidal GaN-NCs with spherical shape within $4.2 \pm 1.9 \mathrm{~nm}$ particle size distribution. XRD and selected area electron diffraction analysis of the product via fs PLA revealed that GaN-NCs are in wurtzite structure. Moreover, X-ray photoelectron spectroscopy measurements also confirm the presence of $\mathrm{GaN}$ nanomaterials. The colloidal GaN-NCs solution exhibits strong blue shift in the absorption
\end{abstract}

\footnotetext{
A. Demirel · İ. Yilmaz $(\bowtie)$

Department of Electrical and Electronics Engineering, Turgut Özal University, 06010 Keçiören, Ankara, Turkey e-mail: iyilmaz@turgutozal.edu.tr

T. Öztaş · C. Kurşungöz · B. Ortaç National Nanotechnology Research Center, Bilkent University, 06800 Bilkent, Ankara, Turkey

C. Kurşungöz $\cdot$ B. Ortaç $(\bowtie)$

Institute of Material Science and Nanotechnology, Bilkent University, 06800 Bilkent, Ankara, Turkey

e-mail: ortac@unam.bilkent.edu.tr
}

spectrum compared to that of the GaN-NPs via ns PLA in ethanol. Furthermore, the photoluminescence emission behavior of fs PLA-generated GaN-NCs in the $295-400 \mathrm{~nm}$ wavelength range is observed with a peak position located at $305 \mathrm{~nm}$ showing a strong blue shift with respect to the bulk $\mathrm{GaN}$.

Keywords Gallium nitride nanoparticles . Femtosecond/nanosecond pulsed laser ablation in organic solution - Ultrasmall nanocrystal generation · Photoluminescence

\section{Introduction}

For the last two decades, III-N-based semiconductors with their wide, direct, and tunable band gap from IR to UV regions $(0.7-6.2 \mathrm{eV})$, their ternary and quaternary alloys have attracted much attention for optoelectronic devices (Akasaki and Amano 1997; Kazlauskas et al. 2003; Dong et al. 2014). GaN-based materials having a high strength to high power, high temperature, high frequency are suitable candidates for devices in harsh environments (Son et al. 2010). Development of GaN-based blue light emission devices (e.g., light-emitting diodes and laser diodes) (Nakamura et al. 1991, 1996; Denbaars 1997) accelerated the research on GaN. By limiting the size of a material to smaller than the doubled exciton Bohr radius, the material starts showing novel optical and structural properties like blue shift in optical 
absorption and PL spectra in comparison with the band gap emission of bulk material (Ramvall et al. 1998; Gyger et al. 2014). Crystalline GaN quantum dots provide enhanced carrier confinement-induced blue shift (Leppert et al. 1998; Mićić et al. 1999; Klimov et al. 2000; Preschilla et al. 2000; Miyamura et al. 2002) and allow one to design core-shell nanostructures (Reiss et al. 2009). GaN QDs are demonstrated high sensitivity in gas sensing, e.g., $\mathrm{H}_{2}$ and ethanol and biocompatible candidate in bio-imaging as luminescence probes (Fan et al. 2014).

A number of nanocrystalline $\mathrm{GaN}$ studies were reported through some grown techniques (Mićić et al. 1999; Borsella et al. 2001; Miyamura et al. 2002; Zhuang et al. 2013; Gyger et al. 2014). GaN quantum dots were grown by Miyamura et al. (2002) through low-pressure metalorganic chemical vapor deposition. Two blue-shifted PL peaks were observed at 4.18 and $3.59 \mathrm{eV}$ for two different quantum dot structures. Hexagonal nanocrystalline $\mathrm{GaN}$ with the size of 2-3 nm was generated by Borsella et al. (2001) via sequential $\mathrm{Ga}$ and $\mathrm{N}$ ions implantation into crystalline and amorphous dielectrics tracked by annealing of the samples $\mathrm{GaN}$ in flowing ammonia gas at $900{ }^{\circ} \mathrm{C}$. Recently, quantum-confined cubic $\mathrm{GaN}$ nanoparticles with the size of 3-4 $\mathrm{nm}$ have been synthesized by Gyger et al. (2014) via liquid-ammonia-in-oil-microemulsions. Laser ablation method is also a promising technique to generate a wide variety of metal and semiconductor nanoparticles (Yang 2012; Alkis et al. 2012; Zeng et al. 2012). Unlike GaN nanostructures obtained by chemical methods (Yang et al. 1999; Sardar and Rao 2004; Ganesh et al. 2010; Chirico and Hector 2010), with laser ablation it is possible to produce chemical-free and clean nanomaterials which can be used in biomedical applications (Petersen and Barcikowski 2009; Wu et al. 2011). Laser ablation can be performed in various environments such as liquid and diluted gas/vacuum. The laser ablation of solids in liquid environment compared to dilute gas or vacuum needs simple setup for the generation of nanoparticles. Moreover, it allows advanced and functional nanoparticle production (Liu et al. 2010; Zeng et al. 2012; Barcikowski and Compagnini 2013). The properties of laser-generated nanoparticles depend on various experimental considerations such as liquid material properties, laser parameters, and laser-material interaction conditions (Mafuné et al. 2000; Barcikowski et al. 2007). A shock wave is created by laser-induced plasma due to the confinement of liquid. This increases the temperature, the pressure, and density of the plasma. A short pulse of laser in nanosecond time domain induces higher plasma-induced pressure. The condensation of the plasma plume due to the cooling down of the confining liquid leads to generation of dispersed small particles into the liquid during the plasma quenching (Berthe et al.1997; Yang 2007, 2012). The short quenching times of the plasma plume can effectively limit the size of the particle grown. In nanosecond pulse regime, electrons are removed from the bulk within $10 \mathrm{ps}$ and oscillating electrons transfer energy to the lattice. Then irradiated surface is heated up and vaporized (Hashida et al. 2009). The ablation process in fs time scale is different than the ns one. Energy deposition via multiphoton absorption to the target is faster than the electron-phonon thermal processes. Therefore, expansion of plasma starts after the fs pulse terminates. Since the nature of the laser material interaction in $\mathrm{ns} / \mathrm{fs}$ time domain is fundamentally different, it is observed that the properties (particle size, crystalline structure etc.) of the product could be different. For example, compared to ns PLA, laser ablation with femtosecond laser may offer smaller particle generation with narrow size distribution (Tsuji et al. 2003; Kabashin and Meunier 2003, Intartaglia et al. 2011). There are few reports on the generation of GaN-NCs through laser ablation technique. Leppert et al. (1998) reported that GaN-NCs were synthesized through PLA of a Ga metal target in nitrogen atmosphere with the post-treatment of annealing at $800{ }^{\circ} \mathrm{C}$ in order to obtain crystalline $\mathrm{GaN}$ nanostructures. Size distribution was found with a mean value of $4.5 \pm 1.6 \mathrm{~nm}$. Due to the quantum confinement effect, blue shifts were observed in both absorption and PL spectra. Liu et al. (2008) synthesized GaN-NCs with the size distribution in the range of $50-150 \mathrm{~nm}$ via nanosecond laser ablation in ethanol. They observed phase transition from hexagonal to hexagonal-cubic crystal structures. Due to large sizes of GaN-NCs quantum confinement, effects were not observed. To our knowledge, there is no study on generation of GaN-NCs by femtosecond laser ablation of solid target in liquid environment.

In this study, firstly sub-25-nm-sized amorphous GaN-NPs in ethanol were synthesized by ns PLA of $\mathrm{GaN}$ powder. Then, the particle size was reduced to sub-10 $\mathrm{nm}$ remaining amorphous structure by the implementation of polymeric matrix. Secondly, sub-8- 
nm-sized GaN-NCs with wurtzite crystalline structure were generated by introducing fs PLA process in ethanol without using any post-treatment or agent. The structural and the optical properties of GaN-NPs generated by ns and fs PLA were studied by TEM and UV/Vis absorption spectroscopy. The direct generation of crystalline and chemical bonding feature of GaN-NCs generated by fs PLA was confirmed using XRD, TEM with selected area electron diffraction (SAED) and XPS techniques. A strong blue shift in photoluminescence (PL) spectra of GaN-NCs was observed resulting from the quantum confinement effects based on their particle sizes smaller than the doubled exciton Bohr radius.

\section{Materials and experimental methods}

The ns PLA of GaN powder was performed using a commercial nanosecond pulsed ND:YLF laser (Empower Q-Switched Laser, Spectra Physics) operated at $527 \mathrm{~nm}$ with pulse duration of $100 \mathrm{~ns}$ and a pulse repetition rate of $1 \mathrm{kHz}$. The laser output power was $16 \mathrm{~W}$ with the pulse energy of $16 \mathrm{~mJ}$. A glass vial was first cleaned by acetone before PLA process. The laser beam was focused on $1 \mathrm{mg}$ bulk $\mathrm{GaN}$ powder (99.99\% Sigma-Aldrich) target placed in the glass vial containing $10 \mathrm{ml}$ of ethanol $(99.8 \%$ SigmaAldrich) using a plano-convex lens with a focal length of $50 \mathrm{~mm}$. The laser ablation was carried out for $60 \mathrm{~min}$. The polymer matrix was prepared by mixing $250 \mathrm{mg}$ of Polyvinylpyrrolidone (PVP) with molecular weight of $15,000 \mathrm{~g} / \mathrm{mol}$ with $10 \mathrm{ml}$ of ethanol. Generation of GaN-NCs was performed utilizing a commercial femtosecond pulsed Ti:Sapphire laser system operated at $800 \mathrm{~nm}$ with pulse duration of $150 \mathrm{fs}$ and average output power of $1.1 \mathrm{~W}$ with a pulse repetition rate of $1 \mathrm{kHz}$ corresponding to a pulse energy of $1.1 \mathrm{~mJ}$. In order to synthesize pure GaN nanocrystals with the sizes of less than the doubled exciton Bohr radius which exhibit quantum confinement effects via single-step technique, we investigated effects of several parameters such as laser parameters and liquid environments. We performed ns PLA in ethanol and in PVP and then fs PLA in ethanol environment to obtain crystalline GaN nanostructures. The laser ablation process took place under the following experimental conditions: the height of liquids layer over the bulk GaN powder target was about $5 \mathrm{~mm}$. To obtain colloidal GaN-NCs solution, the solution was continuously stirred by a magnetic stirrer at $675 \mathrm{rpm}$. The pulsed laser ablation process was carried out for $90 \mathrm{~min}$. For ns and fs experiments, the fluence densities $\left(\mathrm{J} / \mathrm{cm}^{2}\right)$ at the focal points are calculated as 40 and $350 \mathrm{~J} / \mathrm{cm}^{2}$, respectively.

The morphology, the chemical composition analysis, and the crystalline structure of initial material based on bulk GaN powder were performed using a FEI-Quanta 200 FEG SEM instrument equipped with EDAX system and XRD which were carried out in a PANalytical X'Pert PRO MPD diffractometer using $\mathrm{Cu} \mathrm{K} \alpha$ radiation. XRD patterns were obtained in the range of $20-80^{\circ}$ with a step size of $0.1^{\circ}$. On the other hand, the morphology and the crystalline structure of GaN-NPs solution were performed by TEM (FEI-Tecnai G2 F30) instrument at an operating voltage of $300 \mathrm{kV}$ with SAED and XRD (similar condition as shown in GaN powder study above). TEM and XRD samples were prepared by drop-casting solutions onto carbon-coated TEM grids and glass wafer, respectively. The crystalline structure of the NPs was evaluated by XRD measurements. Peak positions and crystallite size values were obtained by fitting the GIXRD data using PANalytical X'Pert HighScore Plus Software. The crystallite size was determined by line profile analysis (LPA) using the same software. In order to obtain surface composition and chemical states of GaN-NPs solution, XPS study was also performed. The XPS data were recorded with GaN-NPs samples placed on quartz substrates. XPS measurements were performed on a monochromatic K-Alpha instrument (Thermo) operating at $12 \mathrm{kV}$ and $2.5 \mathrm{~mA}$. XPS spectra were collected with a photoelectron take-off angle of $90^{\circ}$ from a $200 \mu \mathrm{m}$ diameter circular spot on the sample surface plane, energy steps of $0.1 \mathrm{eV}$, and pass energy of $30 \mathrm{eV}$. The control of the flow of the electrons to the surface was achieved by means of a well-controlled flood gun technique. The optical absorption of ns and fs PLAgenerated products and PL properties of fs PLA-generated GaN-NCs were studied using a Varian Cary 5000 UV/Vis/NIR spectrophotometer in the $200-800 \mathrm{~nm}$ wavelength range and a Varian Cary eclipse fluorescence spectrophotometer in the $295-525 \mathrm{~nm}$ spectral range, respectively. The GaN-NPs samples in ethanol and PVP were prepared in quartz cuvettes. 


\section{Results and discussion}

The GaN powder as starting material was tested before the laser ablation by SEM, EDAX, and XRD. The morphology of the crystalline powder was the platelike form, with micron size and in wurtzite structure. During the laser ablation process, the formation of plasma was observed and the organic solution became light yellow in color for all products. Firstly, we focus on the generation of the GaN-NPs in ethanol under the ns pulsed laser. Figure 1 shows TEM image of GaNNPs generated by nanosecond pulsed laser ablation in ethanol. The GaN-NPs are in spherical shape, mostly less than $20 \mathrm{~nm}$ in size and have amorphous structure. The nanosecond pulses produced large-size distribution of $12.4 \pm 7.0 \mathrm{~nm}$ as displayed in the inset histogram. The size distribution is obtained from
TEM images by considering 150 nanoparticles. The power density in the order of $10^{8} \mathrm{~W} / \mathrm{cm}^{2}$ in our experiment corresponds to the lower limit of the vapor and plasma generation range (Zeng et al. 2012). The resultant NPs are smaller in size compared to the ones in Liu et al. (2008) work in which two orders of magnitude with higher power density applied.

Secondly, we change the liquid environment in ns PLA technique. Similarly, GaN-NPs are produced in polymer matrix via ns PLA. All parameters are kept the same except the liquid environment and the GaNNPs are again in amorphous structure as shown in the SAED pattern in the inset in Fig. 2. However, ns PLA in PVP resulted in amorphous GaN-NPs with size distribution of $6.4 \pm 2.3 \mathrm{~nm}$. As seen in the histogram, the nanoparticles are about the doubled exciton Bohr radius in size. Both the mean value and

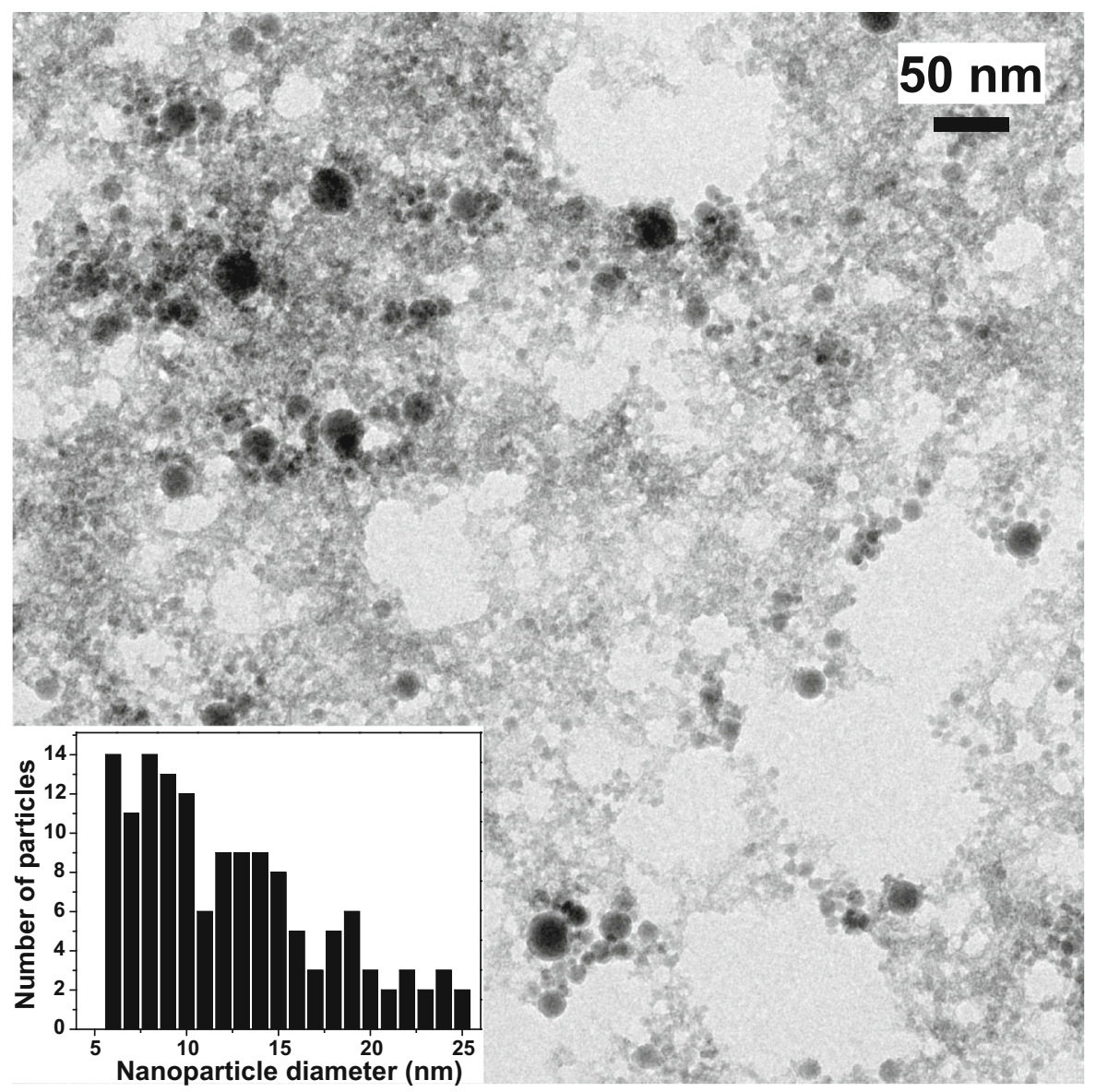

Fig. 1 Representative TEM image of amorphous GaN-NPs generated by nanosecond pulsed laser ablation in ethanol. The inset shows the histogram of size distribution calculated from TEM images 


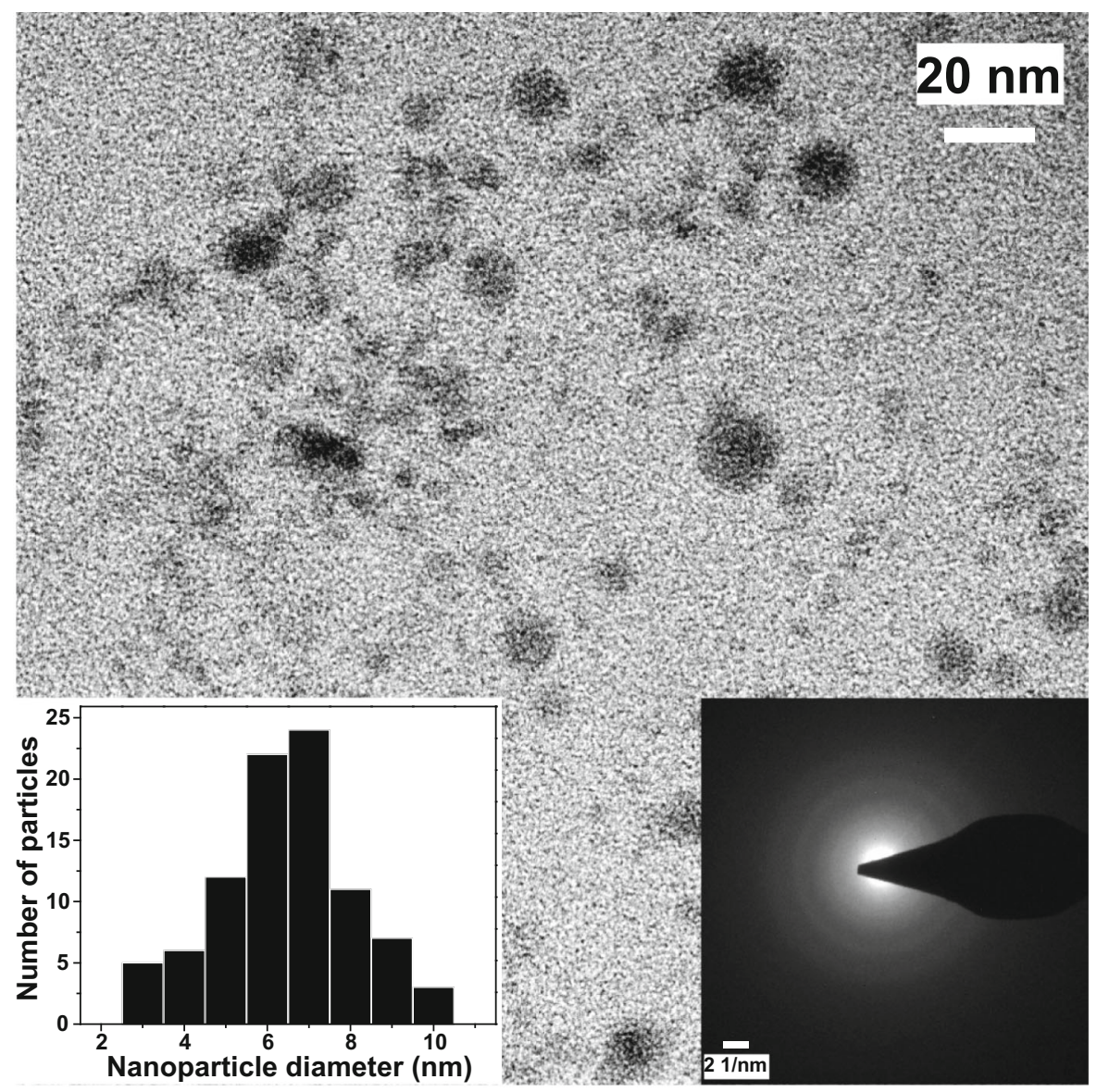

Fig. 2 TEM image of amorphous GaN-NPs generated by nanosecond pulsed laser ablation in PVP. The inset shows the corresponding SAED patterns and the histogram of size distribution calculated from TEM images

standard deviation of the size distribution are diminished when compared with the previous experiment. This smaller and narrower size distribution may be due to the liquid confinement effect. PVP might play a role as surfactant (Mafuné et al. 2002; Kabashin et al. 2003) and provides better control in particle size distribution compared to ethanol only.

Our main motivation is to generate crystalline GaN nanostructures showing quantum confinement effect. Although the above-mentioned trials via ns PLA in ethanol and PVP, the products are in amorphous rather than crystalline structure. Then GaN-NPs are synthesized via fs PLA in ethanol. Figure 3 displays the elemental compositions of the educt and the product obtained by EDAX analysis. The presence of $\mathrm{Ga}$ and $\mathrm{N}$ peaks in the EDAX spectrum of educt confirms that the initial material is based on GaN powder with no significant impurities. However, oxygen and carbon impurities appeared in the EDAX spectrum of the product. These oxygen and carbon impurities may be due to fs PLA process of target GaN powder along with ethanol containing oxygen and carbon atoms. Figure 4 demonstrates XRD patterns of these GaNNPs dispersed on a substrate and the initial GaN powder. The diffraction lines are consistent with the peaks of the hexagonal wurtzite GaN phase (JCPDS card: no. 50-0792) and few $\mathrm{Ga}_{2} \mathrm{O}_{3}$ peaks in beta phase (ICSD collection code: 34243 ). Cubic phase GaN is not observed not only in the final product but also in earlier stages. Similar structural changes depending on laser pulse width have been also observed for GaAs nanoclusters (Trelenberg et al. 2004). When laser pulse width decreases from hundreds of picoseconds to femtosecond regime, the structure of the product alters from near amorphous to crystalline nature. Moreover, GaN-NPs in fs PLA are only in wurtzite 

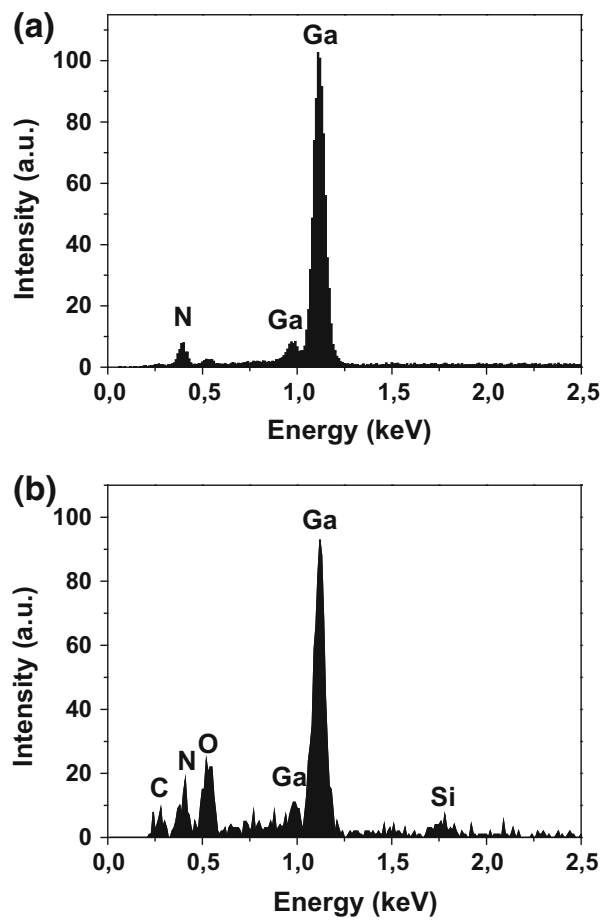

Fig. 3 EDAX analysis of the educt (a) and the product (b) after fs pulsed laser ablation on Si substrate

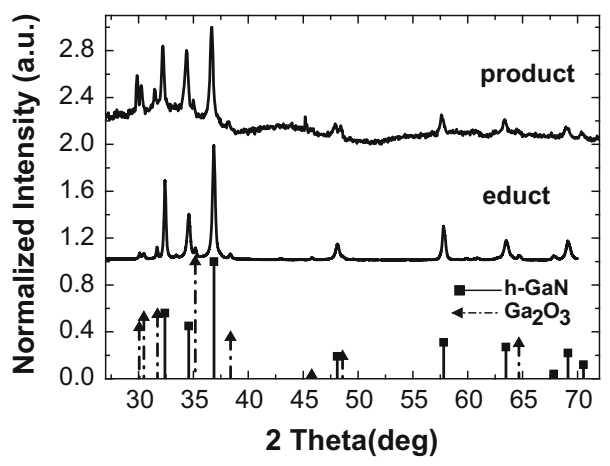

Fig. 4 XRD patterns of the GaN-NPs (product) synthesized by femtosecond pulsed laser ablation (PLA) in ethanol and initial $\mathrm{GaN}$ powder (educt). Squares represent $\mathrm{GaN}$ wurtzite XRD data (JCPDS card: no. 50-0792) and triangles represent few $\mathrm{Ga}_{2} \mathrm{O}_{3}$ peaks (ICSD collection code: 34243 )

structures, both in single and polycrystalline structures but metastable cubic structures (Schaumberg et al. 2015). It shows that along with the ablation, fragmentation (Lau and Barcikowski 2015) might be the main generation mechanism of $\mathrm{GaN}$ nanoparticles from $\mathrm{GaN}$ powder in liquid.
TEM study is then performed to further investigate the structure of GaN-NCs. TEM images of the GaNNCs produced by fs PLA are displayed in Fig. 5. TEM image of the product clearly shows that spherical ultrasmall GaN-NCs are successfully synthesized in a single-step process. TEM studies also indicate that no aggregate well-dispersed nanoparticles are present. The histogram of GaN-NCs obtained from TEM
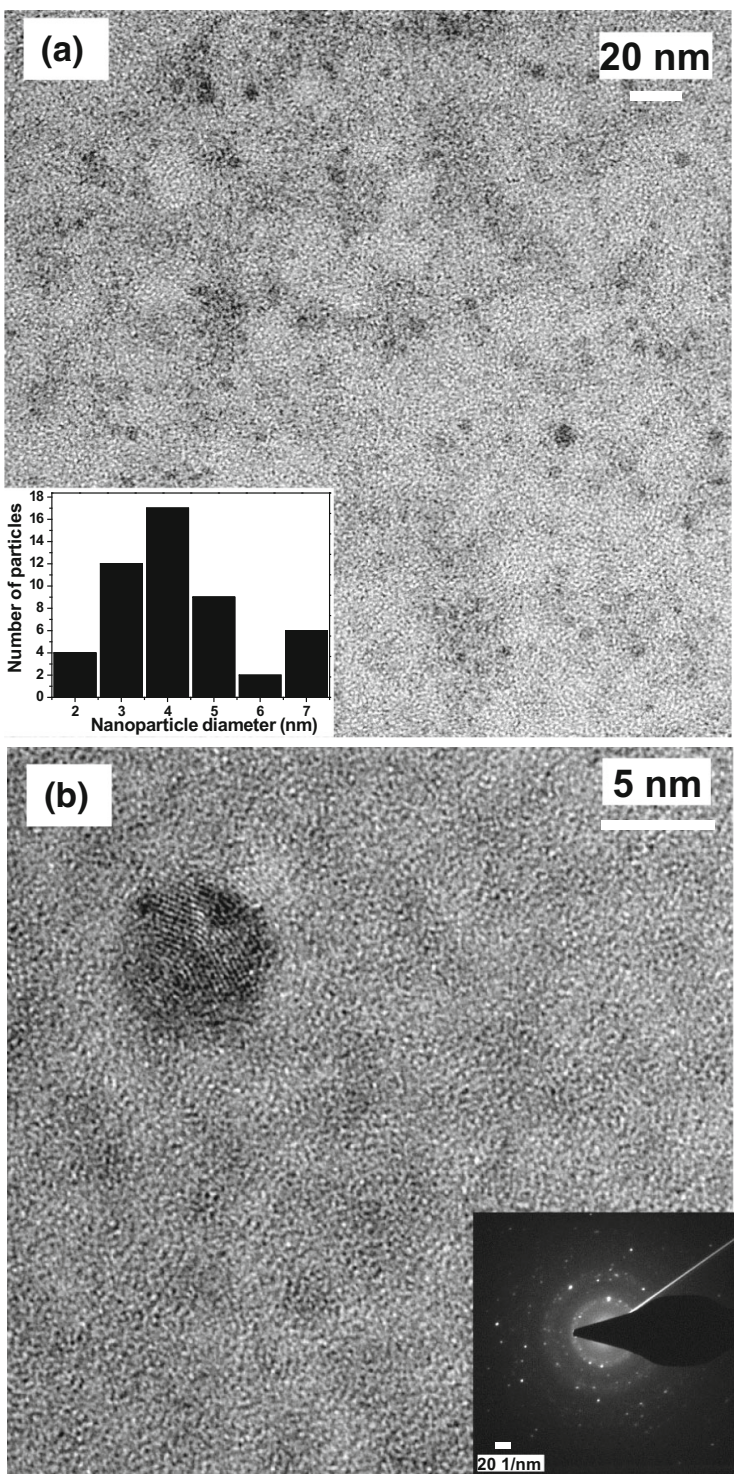

Fig. 5 a Representative TEM image of the GaN-NCs. The inset shows the histogram of size distribution calculated from TEM images. b HRTEM image of a single synthesized GaN-NC showing lattice fringe planes. The inset shows the corresponding SAED pattern 
images (see the inset of Fig. 5a) predicts that the average particle size is $4.2 \pm 1.9 \mathrm{~nm}$ which is smaller than the doubled exciton Bohr radius (Ramvall et al. 1998). It is found that a large portion of the NCs have the diameter below $8 \mathrm{~nm}$. Figure $5 \mathrm{~b}$ presents the highresolution TEM (HRTEM) image of a GaN-NC. HRTEM image of the single isolated GaN-NC presents the crystalline lattice fringes which also confirm GaN-NCs generation by fs PLA technique in ethanol. The inset of Fig. 5b displays SAED analysis of the GaN-NCs product. The calculated d-spacing values $(2.763,2.599,2.442,1.892,1.598,1.467$, $1.384,1.360$, and $1.334 \AA$ ) from the SAED pattern are matching with hexagonal wurtzite structure (JCPDS card: no. 50-0792).

The elemental composition and the chemical state of the GaN-NCs were studied by XPS technique. Figure 6 shows the XPS spectra of the Ga $2 p_{3 / 2}$, Ga $2 \mathrm{p}_{1 / 2}$, and $\mathrm{N} 1 \mathrm{~s}$ spectral core region of a representative GaN-NCs sample. From XPS data, Ga $2 p_{3 / 2}, G a p_{1 / 2}$, and $\mathrm{N} 1 \mathrm{~s}$ core level peak positions were obtained at 1118.2, 1144.8, and $399.4 \mathrm{eV}$, respectively. These results well agree with the literature (Wolter et al.
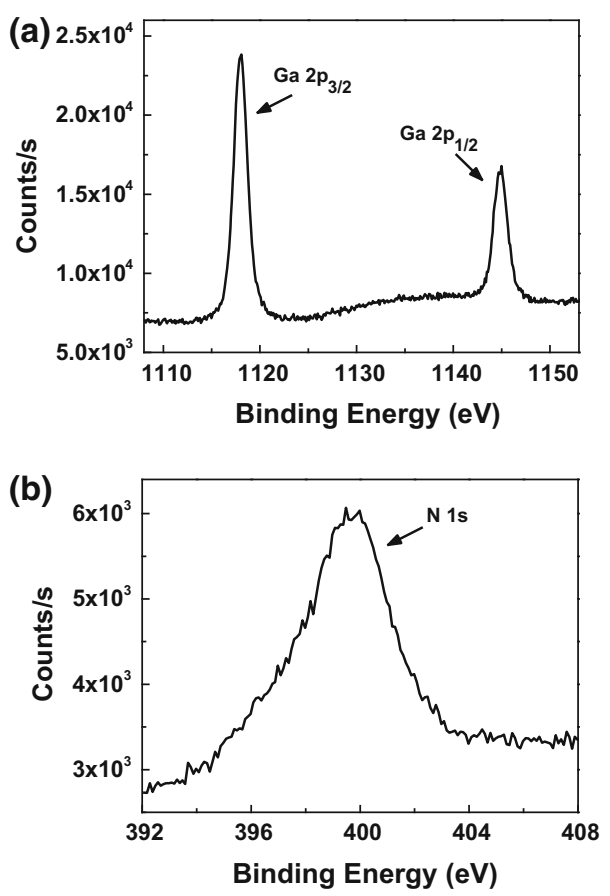

Fig. 6 XPS spectra of a Ga $2 p_{3 / 2}$ and Ga $2 p_{1 / 2}$, b N 1 s of representative $\mathrm{GaN}-\mathrm{NCs}$ sample
1997; Pal et al. 2003) confirming the presence of $\mathrm{GaN}$ nanomaterials.

The optical properties of ns/fs PLA-generated GaN have been characterized by UV/Vis absorption and PL spectroscopy techniques. Figure 7 demonstrates the normalized, room temperature optical absorption spectra of GaN-NPs via fs and ns PLA in ethanol. GaN-NPs via ns PLA exhibits typical absorption spectra of amorphous GaN-NPs with the band gap range of 2.8-3.95 eV (Stumm and Drabold 1997; Bittar et al. 2001; Grigorescu et al. 2003). On the other hand, the optical absorption spectrum of GaN-NPs via fs PLA shows strong absorption behavior starting from $285 \mathrm{~nm}(4.35 \mathrm{eV})$ to shorter wavelengths (i.e., higher energies). This large blue shift of $\sim 1 \mathrm{eV}$ in the optical absorption spectrum compared to bulk GaN material indicates a reduction in nano-sized range occurring due to quantum size effects of the carrier confinement (Leppert et al. 1998; Mićić et al. 1999; Bagga et al. 2003; Li and Wang 2005).

In order to obtain PL spectra, the colloidal GaNNCs solution was excited by 280 and $300 \mathrm{~nm}$ wavelength light. The PL spectra of GaN-NCs in ethanol are shown in Fig. 8. Firstly, by $280 \mathrm{~nm}$ excitation, PL emission in the 295-400 nm wavelength range was observed with the peak position located at $305 \mathrm{~nm}(4.06 \mathrm{eV})$. The peak located at $4.06 \mathrm{eV}$ in PL spectrum indicates a large blue shift $(0.66 \mathrm{eV})$ of band gap emission compared to the bandgap of the bulk GaN crystal (i.e., $3.40 \mathrm{eV}$ ). The large blue shift on the PL emission implies strong quantum confinement effects in GaN-NCs due to ultrasmall NCs generation (i.e., smaller than the doubled exciton Bohr radius for GaN (Ramvall et al.

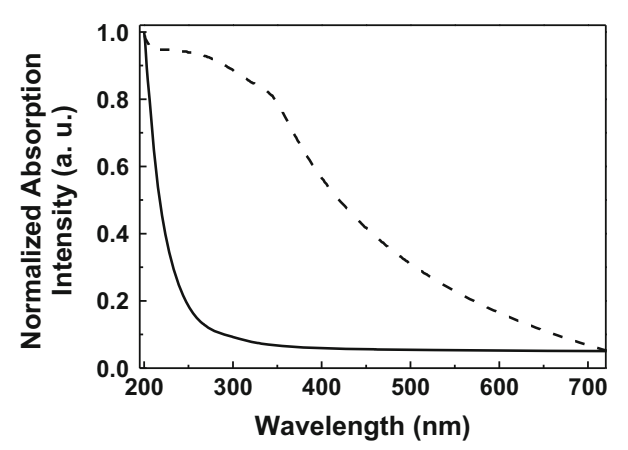

Fig. 7 UV-Vis normalized absorption spectra of GaN-NPs generated by fs (solid line) and ns (dashed line) PLA in ethanol solution 


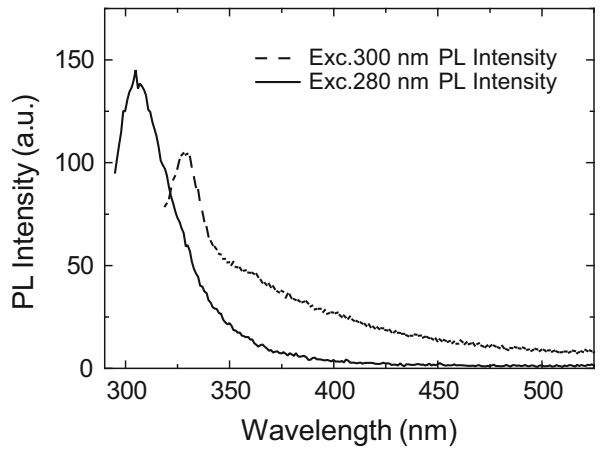

Fig. 8 Photoluminescence spectra of GaN-NCs excited at 280 and $300 \mathrm{~nm}$

1998) using fs PLA (Miyamura et al. 2002; Mazumder and Hector 2008). Gyger et al. (2014) observed blue shift of $1.1 \mathrm{eV}$ in PL spectra of 3-4 nm size GaN-NCs compared to band gap energy of bulk $\mathrm{GaN}$. When $\mathrm{GaN}$ crystals become clusters, there are several effects which may change the band gap according to some theoretical studies and calculations (Brus 1986; Kayanuma 1988; Yoffe 2002; Dimos et al. 2012). In the literature, there are several theoretical methods for nanocrystal studies such as $\vec{k} . \vec{p}$ method (Bagga et al. 2003), tight-binding method (Echeverría-Arrondo et al. 2008), empirical pseudopotential method, and DFT method (Li and Wang 2005). In order to understand our observation, we compared size dependence of exciton energies of $\mathrm{GaN}$ quantum dots (see Fig. 22 in ref. Li and Wang 2005) with our particle size and observed blue shift of $0.66 \mathrm{eV}$. This blue shift is close to the value of $0.5 \mathrm{eV}$ in ref. Li and Wang 2005 calculations by ab initio DFT method. Moreover, according to the effective mass approximation (EMA) (Brus 1986) but excluding Coulomb term and polarization term, $\Delta E=\frac{\hbar^{2} \pi^{2}}{2 \mu R^{2}}$, the blue shift in our case is calculated as $0.558 \mathrm{eV}$ (The effective mass values are taken from the ref. Echeverría-Arrondo et al. 2008). However, we observed the blue shift of $0.66 \mathrm{eV}$. When we calculate the radius of the nanoparticle from this shift, it corresponds to the radius of $2.0 \mathrm{~nm}$ which is very close to the half of the mean size (diameter) of $4.4 \mathrm{~nm}$ from the histogram of the our nanoparticle size distribution. Furthermore, in our PL spectra, we have not observed defect-related blue band emission as seen in some references (Kumar et al. 2010; Reshchikov and Morkoç 2005). Second, by $300 \mathrm{~nm}$ excitation, the PL emission peak moves to
$329 \mathrm{~nm}(3.769 \mathrm{eV})$. This peak may be attributed to band to band transition for $6.5 \mathrm{~nm}$ size GaN-NCs (Bagga et al. 2003; Echeverría-Arrondo et al. 2008). This wavelength excitation-dependent photoluminescence may be due to the size distribution of GaN-NCs (Cao et al. 2000). Photons at $300 \mathrm{~nm}$ cannot excite the GaN-NCs with band gap energy of larger than $4.13 \mathrm{eV}$. Therefore, the emission wavelength shift is expected with excitation wavelength (Xi-Feng et al. 2008). These results display that quantum confinement effect plays a key role on the optical properties (absorption and PL) of these GaN-NC quantum dots.

\section{Conclusion}

In conclusion, we demonstrated the generation of ultrasmall GaN-NCs through a one-step femtosecond laser ablation in ethanol. The colloidal GaN-NCs were successfully synthesized with spherical shape within $4.2 \pm 1.9 \mathrm{~nm}$ particle size distribution which is smaller than the doubled exciton Bohr radius. Hexagonal wurtzite crystalline structure was then verified with XRD data and the SAED pattern. The XPS study also confirmed GaN nanostructure synthesis. The structural characterization of GaN-NPs via ns PLA in ethanol and PVP revealed that amorphous GaN-NPs are synthesized with the smaller and narrower size distribution of $6.4 \pm 2.3 \mathrm{~nm}$ in PVP than the size distribution of $12.4 \pm 7.0 \mathrm{~nm}$ in ethanol. The UV/Vis spectrum analysis showed that the absorption edge shifted by $1 \mathrm{eV}$ to the shorter wavelength and the PL peak at $4.06 \mathrm{eV}$ with the blue shift of $0.66 \mathrm{eV}$ compared to bulk GaN were observed from GaNNCs via fs PLA due to the quantum confinement effect. Because of their promising optical absorption and PL properties, these $\mathrm{GaN}$ nanomaterials could be prime candidates for bio and optical device applications such as biosensors and photodetectors.

Acknowledgments This work is supported by the Scientific Research Fund of Turgut Özal University under the project number of 001-04-2012. State Planning Organization (DPT) of Turkey is acknowledged for the support of UNAM-Institute of Materials Science and Nanotechnology. B.O. acknowledges partial support of TUBITAK (Project No: 113S223), The Turkish Academy of Sciences-Outstanding Young Scientists Award Program (TUBA-GEBIP), Bilim Akademisi-The Science Academy, Turkey under the BAGEP program, and METU Prof. Dr. Mustafa Parlar Foundation. 


\section{References}

Akasaki I, Amano H (1997) Crystal growth and conductivity control of group III nitride semiconductors and their application to short wavelength light emitters. Jpn J Appl Phys 36:5393-5408. doi:10.1143/JJAP.36.5393

Alkis S, Alevli M, Burzhuev S, Vural HA, Okyay AK, Ortaç B (2012) Generation of InN nanocrystals in organic solution through laser ablation of high pressure chemical vapor deposition-grown InN thin film. J Nanopart Res 14:1048. doi:10.1007/s11051-012-1048-5

Bagga A, Chattopadhyay PK, Ghosh S (2003) Energy levels of nitride quantum dots: wurtzite versus zinc-blende structure. Phys Rev B 68:155331. doi:10.1103/PhysRevB.68. 155331

Barcikowski S, Compagnini G (2013) Advanced nanoparticle generation and excitation by lasers in liquids. Phys Chem Chem Phys 15:3022-3026. doi:10.1039/c2cp90132c

Barcikowski S, Hahn A, Kabashin AV, Chichkov BN (2007) Properties of nanoparticles generated during femtosecond laser machining in air and water. Appl Phys A 87:47-55. doi:10.1007/s00339-006-3852-1

Berthe L, Fabbro R, Peyre P, Tollier L, Bartnicki E (1997) Shock waves from a water-confined laser-generated plasma. J Appl Phys 82:2826-2832. doi:10.1063/1.366113

Bittar A, Trodahl HJ, Kemp NT, Markwitz A (2001) Ion-assisted deposition of amorphous GaN: Raman and optical properties. Appl Phys Lett 78:619-621. doi:10.1063/1. 1345800

Borsella E, Garcia MA, Mattei G et al (2001) Synthesis of GaN quantum dots by ion implantation in dielectrics. J Appl Phys 90:4467-4473. doi:10.1063/1.1408591

Brus L (1986) Electronic wave functions in semiconductor clusters: experiment and theory. $\mathrm{J}$ Phys Chem 90:2555-2560. doi:10.1021/j100403a003

Cao YG, Chen XL, Li JY, Lan YC, Liang JK (2000) Observation of a quantum-confinement effect with $\mathrm{GaN}$ nanoparticles synthesized through a new gas reaction route. Appl Phys A 71:229-231. doi:10.1007/s003390000560

Chirico P, Hector AL (2010) Solvothermal synthesis of gallium and indium nitrides using lithium amide. $Z$ Naturforschung 65:1051-1057. doi:10.1515/znb-2010-0812

Denbaars SP (1997) Gallium-nitride-based materials for blue to ultraviolet optoelectronics devices. Proc IEEE 85:1740-1749. doi:10.1109/5.649651

Dimos K, Jankovic L, Koutselas IB, Karakassides MA, Zboril R, Komadel P (2012) Low-temperature synthesis and characterization of gallium nitride quantum dots in ordered mesoporous silica. J Phys Chem C 116:1185-1194. doi:10. 1021/jp208011y

Dong P, Yan J, Zhang Y et al (2014) Optical properties of nanopillar AlGaN/GaN MQWs for ultraviolet light-emitting diodes. Opt Express 22:320-327. doi:10.1364/OE.22.00A320

Echeverría-Arrondo C, Pérez-Conde J, Bhattacharjee AK (2008) Acceptor and donor impurities in GaN nanocrystals. J Appl Phys 104:044308. doi:10.1063/1.2970165

Fan G, Wang C, Fang J (2014) Solution-based synthesis of III-V quantum dots and their applications in gas sensing and bioimaging. Nano Today 9:69-84. doi:10.1016/j.nantod.2014. 02.007
Ganesh V, Suresh S, Balaji M, Baskar K (2010) Synthesis and characterization of nanocrystalline gallium nitride by nitridation of Ga-EDTA complex. J Alloy Compd 498:52-56. doi:10.1016/j.jallcom.2010.03.068

Grigorescu CEA, Lee HK, Lanke UD et al (2003) Annealing amorphous $\mathrm{GaN}$ - a way to nano-crystalline state. In: Proceedings of 2003 5th international conference on transparent optical networks, vol 1, pp 337-339. doi:10. 1109/ICTON.2003.1264649

Gyger F, Bockstaller P, Gröger H, Gerthsen D, Feldmann C (2014) Quantum-confined GaN nanoparticles synthesized via liquid-ammonia-in-oil-microemulsions. Chem Commun 50:2939-2942. doi:10.1039/c4cc00180

Hashida M, Mishima H, Tokita S, Sakabe S (2009) Non-thermal ablation of expanded polytetrafluoroethylene with an intense femtosecond-pulse laser. Opt Express 17:13116-13121. doi:10.1364/OE.17.013116

Intartaglia R, Bagga K, Brandi F et al (2011) Optical properties of femtosecond laser-synthesized silicon nanoparticles in deionized water. J Phys Chem C 115:5102-5107. doi:10. 1021/jp109351t

Kabashin AV, Meunier M (2003) Synthesis of colloidal nanoparticles during femtosecond laser ablation of gold in water. J Appl Phys 94:7941-7943. doi:10.1063/1.1626793

Kabashin AV, Meunier M, Kingston C, Luong JHT (2003) Fabrication and characterization of gold nanoparticles by femtosecond laser ablation in an aqueous solution of cyclodextrins. J Phys Chem B 107:4527-4531. doi:10. 1021/jp034345q

Kayanuma Y (1988) Quantum-size effects of interacting electrons and holes in semiconductor microcrystals with spherical shape. Phys Rev B 38:9797. doi:10.1103/ PhysRevB.38.9797

Kazlauskas K, Tamulaitis G, Zukauskas A et al (2003) Exciton and carrier motion in quaternary AlInGaN. Appl Phys Lett 82:4501-4503. doi:10.1063/1.1586782

Klimov VI, Mikhailovsky AA, Xu S et al (2000) Optical gain and stimulated emission in nanocrystal quantum dots. Science 290:314-317. doi:10.1126/science.290.5490.314

Kumar P, Panchakarla LS, Bhat SV, Maitra U, Subrahmanyam KS, Rao CNR (2010) Photoluminescence, white light emitting properties and related aspects of $\mathrm{ZnO}$ nanoparticles admixed with graphene and GaN. Nanotechnology 21:385701. doi:10.1088/0957-4484/21/38/385701

Lau M, Barcikowski S (2015) Quantification of mass-specific laser energy input converted into particle properties during picosecond pulsed laser fragmentation of zinc oxide and boron carbide in liquids. Appl Surf Sci 348:22-29. doi:10. 1016/j.apsusc.2014.07.053

Leppert VJ, Zhang CJ, Lee HW, Kennedy IM, Risbud SH (1998) Observation of quantum confined excited states of $\mathrm{GaN}$ nanocrystals. Appl Phys Lett 72:3035-3037. doi:10.1063/ 1.121532

Li J, Wang LW (2005) Band-structure-corrected local density approximation study of semiconductor quantum dots and wires. Phys Rev B 72:125325. doi:10.1103/PhysRevB.72.125325

Liu P, Cao YL, Cui H, Chen XY, Yang GW (2008) Synthesis of GaN nanocrystals through phase transition from hexagonal to cubic structures upon laser ablation in liquid. Cryst Growth Des 8:559-563. doi:10.1021/cg0705963 
Liu P, Cui H, Wang CX, Yang GW (2010) From nanocrystal synthesis to functional nanostructure fabrication: laser ablation in liquid. Phys Chem Chem Phys 12:3942-3952. doi:10.1039/b918759f

Mafuné F, Kohno J, Takeda Y, Kondow T, Sawabe H (2000) Formation and size control of silver nanoparticles by laser ablation in aqueous solution. J Phys Chem B 104:9111-9117. doi:10.1021/jp001336y

Mafuné F, Kohno J, Takeda Y, Kondow T (2002) Full physical preparation of size-selected gold nanoparticles in solution: laser ablation and laser-induced size control. J Phys Chem B 106:7575-7577. doi:10.1021/jp020577y

Mazumder B, Hector AL (2008) Synthesis and applications of nanocrystalline nitride materials. $J$ Mater Chem 19:4673-4686. doi:10.1039/b817407e

Mićić OI, Ahrenkiel SP, Bertram D, Nozik A (1999) Synthesis, structure, and optical properties of colloidal GaN quantum dots. Appl Phys Lett 75:478-480. doi:10.1063/1.124414

Miyamura M, Tachibana K, Arakawa Y (2002) High-density and size-controlled $\mathrm{GaN}$ self-assembled quantum dots grown by metalorganic chemical vapor deposition. Appl Phys Lett 80:3937-3939. doi:10.1063/1.1482416

Nakamura S, Mukai T, Senoh M (1991) High-power GaN P-N junction blue-light-emitting diodes. Jpn J Appl Phys 30:1998-2001. doi:10.1143/JJAP.30.L1998

Nakamura S, Senoh M, Nagahama S et al (1996) Ridge-geometry InGaN multi-quantum-well-structure laser diodes. Appl Phys Lett 69:1477-1479. doi:10.1063/1.116913

Pal S, Mahapatra R, Ray SK et al (2003) Microwave plasma oxidation of gallium nitride. Thin Solid Films 425:20-23. doi:10.1016/S0040-6090(02)01055-6

Petersen S, Barcikowski S (2009) In situ bioconjugation: single step approach to tailored nanoparticle-bioconjugates by ultrashort pulsed laser ablation. Adv Funct Mater 19:1167-1172. doi:10.1002/adfm.200801526

Preschilla AN, Major S, Kumar N, Samajdar I, Srinivasa RS (2000) Nanocrystalline gallium nitride thin films. Appl Phys Lett 77:1861-1863. doi:10.1063/1.1311595

Ramvall P, Tanaka S, Nomura S, Riblet P, Aoyagi Y (1998) Observation of confinement-dependent exciton binding energy of GaN quantum dots. Appl Phys Lett 73:1104-1106. doi:10.1063/1.122098

Reiss P, Protière M, Li L (2009) Core/shell semiconductor nanocrystals. Small 5:154-168. doi:10.1002/smll. 200800841

Reshchikov MA, Morkoç H (2005) Luminescence properties of defects in GaN. J Appl Phys 97:061301. doi:10.1063/1. 1868059

Sardar K, Rao CNR (2004) New solvothermal routes for GaN nanocrystals. Adv Mater 16:425-429. doi:10.1002/adma. 200306050

Schaumberg CA, Wollgarten M, Rademann K (2015) Fragmentation mechanism of the generation of colloidal copper(I) iodide nanoparticles by pulsed laser irradiation in liquids. Phys Chem Chem Phys 17:17934. doi:10.1039/ c5cp01153a

Son KA, Liao A, Lung G et al (2010) GaN-based high-temperature and radiation-hard electronics for harsh environments. Nanosci Nanotechnol Lett 2:89-95. doi:10.1166/ nnl.2010.1063

Stumm P, Drabold DA (1997) Can amorphous gan serve as a useful electronic material? Phys Rev Lett 79:677-680. doi:10.1103/PhysRevLett.79.677

Trelenberg TW, Dinh LN, Saw CK, Stuart BC, Balooch M (2004) Femtosecond pulsed laser ablation of GaAs. Appl Surf Sci 221:364-369. doi:10.1016/S01694332(03)00937-1

Tsuji T, Kakita T, Tsuji M (2003) Preparation of nano-size particles of silver with femtosecond laser ablation in water. Appl Surf Sci 206:314-320. doi:10.1016/S01694332(02)01230-8

Wolter SD, Luther BP, Waltemyer DL et al (1997) X-ray photoelectron spectroscopy and X-ray diffraction study of the thermal oxide on gallium nitride. J Appl Phys Lett 70:2156-2158. doi:10.1063/1.118944

Wu H, Yang R, Song B et al (2011) Biocompatible inorganic fullerene-like molybdenum disulfide nanoparticles produced by pulsed laser ablation in water. ACS Nano 5:1276-1281. doi:10.1021/nn102941b

Xi-Feng Y, Zhao-Lin L, Ping-Ping C, Xiao-Shuang C, Tian-Xin L, Wei L (2008) Broadening of photoluminescence by nonhomogeneous size distribution of self-assembled InAs quantum dots. Chin Phys Lett 25:3059. doi:10.1088/0256$307 X / 25 / 8 / 087$

Yang GW (2007) Laser ablation in liquids: applications in the synthesis of nanocrystals. Prog Mater Sci 52:648-698. doi:10.1016/j.pmatsci.2006.10.016

Yang GW (2012) Laser ablation in liquids: principles and applications in the preparation of nanomaterials. Pan Standford, Singapore

Yang Y, Leppert VJ, Risbud SH et al (1999) Blue luminescence from amorphous $\mathrm{GaN}$ nanoparticles synthesized in situ in a polymer. Appl Phys Lett 74:2262-2264. doi:10.1063/1. 123819

Yoffe AD (2002) Low-dimensional systems: quantum size effects and electronic properties of semiconductor microcrystallites (zero-dimensional systems) and some quasitwo-dimensional systems. Adv Phys 51:799-890. doi:10. 1080/0001873011011745

Zeng HB, Du XW, Singh SC (2012) Nanomaterials via laser ablation/irradiation in liquid: a review. Adv Funct Mater 22:1333-1353. doi:10.1002/adfm.201102295

Zhuang NF, Wang X, Fei F et al (2013) Mild-temperature synthesis and first-principle fluorescence simulation of GaN nanoparticles. J Nanopart Res 15:1458. doi:10.1007/ s11051-013-1458-z 